\title{
Dark matter with ultra compact mini halos
}

\section{Ki-Young Choi*}

Korea Astronomy and Space Science Institute, Daejeon 305-348, Korea

E-mail: kiyoungchoi@kasi.re.kr

\section{Jinn-Ouk Gong}

Asia Pacific Center for Theoretical Physics, Pohang, 790-784, Korea

Department of Physics, Postech, Pohang 790-784, Korea

E-mail: jinn-ouk.gong@apctp.org

\section{Chang Sub Shin}

Department of Physics and Astronomy, Rutgers University, Piscataway NJ 08854, USA

E-mail: changsub@physics.rutgers.edu

\begin{abstract}
The isocurvature mode of dark matter is not damped away during kinetic decoupling from the thermal plasma. For weakly interaction massive particle as dark matter, the large density perturbation can generate the small scale ultra compact mini halos and the annihilation of dark matter inside them can give accessible signatures in the indirect detection of dark matter. The early matter domination epoch is one way to produce the isocurvature perturbation of weakly interacting massive dark matter at small scales from the initial adiabatic perturbation form single inflation model.
\end{abstract}

18th International Conference From the Planck Scale to the Electroweak Scale 25-29 May 2015

Ioannina, Greece

${ }^{*}$ Speaker. 


\section{Introduction}

Weakly interacting massive particles (WIMPs) are good candidate for dark matter component. WIMPs are initially relativistic and stayed in the thermal equilibrium with the background plasma and they decoupled later due to the expansion of the Universe. Therefore the perturbation of dark matter is inherently adiabatic, which means that the density perturbation of WIMP dark matter follows that of the radiation from where the dark matter came. That is consistent with the observation from the cosmic microwave background (CMB) anisotropy.

While the observations of CMB and large scale structure (LSS) strictly constrains the amplitude of the primordial power spectrum at large scales, there is no observed constraints on the smaller scales. However there is a general upper bound from primordial black hole arguments $[2,3,4,5,6]$. For the scales below the damping of the acoustic modes, the acoustic modes can diffuse and thermalize and put a constraint on the amplitude of primordial fluctuations for certain scales [7].

When the primordial perturbation is large enough at small scales, it can give new signals in the present Universe. Especially when the WIMPs are the dominant component of dark matter, they comprise the main component of the small scale objects such as ultra compact mini halos. Once they are formed, the annihilation of dark matter inside the objects can produce gamma-rays, cosmic rays and neutrinos. However up to now there is no observation of anomalous signals from indirect detection and that sets the upper bound on the primordial density perturbation of WIMP dark matter [9].

The formation of small scale objects can happen also from the large isocurvature perturbation of dark matter on small scales, which is consistent with the adiabaticity on large scales [1]. For WIMP, the perturbation at small scales less than the kinetic decoupling is damped away. However that is only true for adiabatic mode and the isocurvature is not damped and can be large enough. Recently Choi, Gong, and Shin found that the isocurvature perturbation can be generated during the early matter domination epoch and is not damped away even in the kinetic equilibrium with thermal radiation [10].

In this talk, we will sketch the mechanism of the generation of the isocurvature perturbation and show the impact on the observational signatures.

\section{Adiabatic and isocurvature perturbation of dark matter}

We can divide the perturbation of dark matter into adiabatic component with,

$$
\delta_{m}^{a d}=\frac{3}{4} \delta_{r}
$$

and the isocurvature perturbation defined as

$$
S \equiv 3 H\left(\frac{\delta \rho_{m}}{\dot{\rho}_{m}}-\frac{\delta \rho_{r}}{\dot{\rho}_{r}}\right)=\delta_{m}-\frac{3}{4} \delta_{r}
$$

During radiation domination epoch, when the dark matters are still in the kinetic equilibrium with radiation, the adiabatic mode of dark matter perturbation on sub horizon scales follows the oscillations of the radiation and experiences the collisional damping during the kinetic decoupling. The 
suppression of the smaller scales than the kinetic decoupling is considered to give a lower bound for the structure formation at small scales [11, 12, 13].

However it is not true for the isocurvature mode of dark matter. The evolution of the isocurvature perturbation is given by [14]

$$
\dot{S}=\theta_{r}-\theta_{m},
$$

where $\theta_{m}$ and $\theta_{r}$ are the divergence of the velocity of dark matter and radiation respectively. During the kinetic equilibrium, DM is tightly coupled to the radiation, and thus the divergence of the velocity are roughly the same for dark matter and radiation, $\theta_{r}=\theta_{m}$. Therefore we can see easily that the isocurvatutre component remains constant and does not oscillate even in the kinetic equilibrium with radiation.

This property of isocurvature perturbation was used in the past for baryon isocurvature perturbation to explain the large structure formation [15]. However now it is ruled out by the precise observation of the acoustic peaks of the cosmic microwave background (CMB) temperature anisotropy though.

\section{Generation of the isocurvature perturbation of WIMP}

In the standard model of cosmology, the WIMP as well as baryons are in the thermal equilibrium with relativistic particles in the early Universe and then decoupled later. In this case the perturbation of WIMP and baryons are exactly the same as that of their mother particles, thus adiabatic. However there is a situation when the isocurvature perturbation is generated for dark matter in the non-standard cosmology. One of the case is when the dark matter freeze-out during the epoch of the early matter domination prior to the radiation domination [10].

WIMP has weak interaction with the ordinary particles and thus they are in the thermal equilibrium in the early Universe until they become non-relativistic. Once the WIMPs are non-relativistic, sine the temperature of the background becomes smaller than the mass of WIMP, the production of WIMP is significantly reduced and only the annihilation of WIMP occurs, which soon stops too due to the expansion of the Universe. This is why the relic density of the WIMP is determined by the annihilation cross section of them [16] and the freeze-out temperature is around $M_{\mathrm{dm}} / 20$ [13]. After chemical decoupling, the number density of the WIMP decreases only by the expansion of the Universe and therefore the comoving number density preserves. However through the elastic scattering of WIMP with relativistic particles, the WIMPs could till maintain the kinetic equilibrium with radiation by exchanging the momentum each other until later time.

In many non-standard cosmological model, or even in the standard inflationary model, there appears often the early matter domination epoch before the radiation domination. Those include the the inflaton oscillation, heavy partilce domination such as moduli, curvaton, gravitino, or axino [17, 18, 19, 20]. The relativistic particles are produced again from the decay of the heavy particles which dominated before and soon the standard radiation domination begins with a given reheating temperature.

When the reheating temperature is low enough than the freeze-out temperature of the WIMP dark matter, the dark matter freeze-out during matter domination. After that the dominating matter decays and produce radiation. If dark matter production is negligible form the direct decay, the dark 


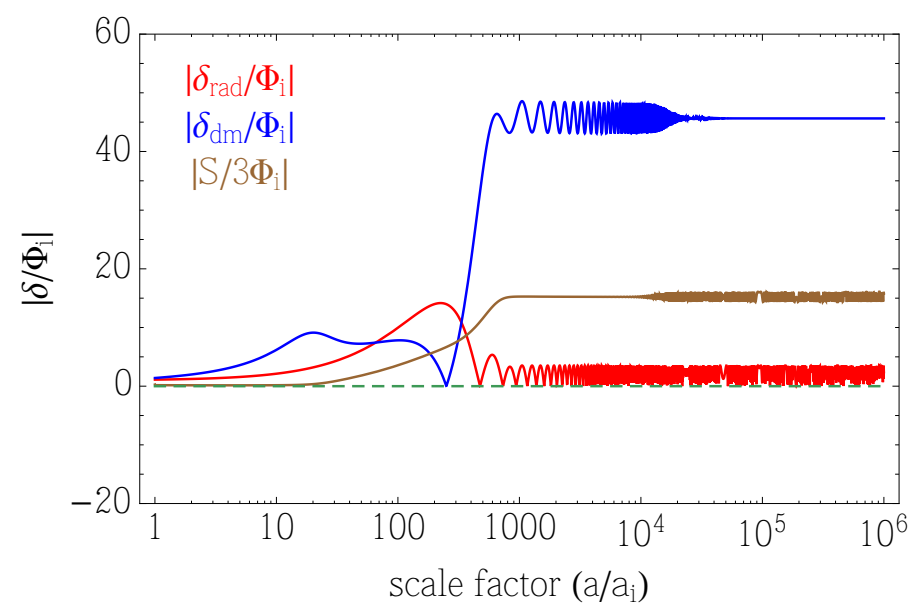

Figure 1: The evolution of the density contrast of the radiation (red), DM (blue) and the isocurvature perturbation (brown) with respect to the initial gravitational potential for $k_{\mathrm{fr}}^{-1}<k^{-1}<k_{\mathrm{reh}}^{-1}$. Figure is adopted from [10].

matters are not produced any more after freeze-out, while the radiation is still produced from the decay of the heavy particle. Between the freeze-out and the reheating, the isocurvature of WIMP dark matter can be generated and estimated as [10]

$$
S\left(t_{\mathrm{reh}}\right) \approx-\frac{3}{4} \int_{t_{i}}^{t_{\mathrm{reh}}} d t \frac{\Gamma_{\phi} \rho_{\phi} \delta_{\phi}}{\rho_{r}} \approx \frac{5}{4} \Phi_{i}\left(\frac{k}{k_{\mathrm{reh}}}\right)^{2}
$$

where $t_{i}$ and $t_{\text {reh }}$ are the initial and final time of the integration and $\Gamma_{\phi}$ is the decay rate of the heavy particle $\phi, \rho_{\phi}, \rho_{r}$ are the energy density of heavy particle and radiation, with $\delta_{\phi}=\delta \rho_{\phi} / \rho_{\phi}$. After integration, $S\left(t_{\text {reh }}\right)$ is roughly given by the gravitational potential at initial time and the ratio of the given scale $k$ and the scale at the reheating $k_{\text {reh }}$, as shown in the last equation.

In Fig. 1, we show the evolution of the density contrast $\delta \equiv \delta \rho / \rho$ for dark matter (blue), radiation (red) and isocurvature perturbation $S$ (brown) normalised by the initial gravitational potential for a scale which is larger than the freeze-out but smaller than the reheating. Here the mode enters the horizon after freeze-out of the WIMP dark matter at around the scale factor $a \simeq 100 a_{i}$. After horizon entry, the perturbation of the radiation oscillates while the dark matter perturbation increases until reheating completes at around the scale factor $a \simeq 500 a_{i}$. The isocurvature perturbation, which is dominant component of dark matter at this scale, is constant and only the adiabatic component oscillates following that of radiation. The oscillation finally decreases and disappears during the kinetic decoupling at around the scale factor $a \simeq 2 \times 10^{4} a_{i}$ since the adiabatic mode is damped away.

\section{Dark matter and the ultra compact mini halos}

When the density perturbation is large enough, they can collapse much earlier than the last scattering moment or just right after the moment into the primordial black hole or ultra compact 
mini halo (UCMH). The UCMH has a steep DMdensity profile from pure radial infall and grows after radiation-matter equality by including DM and baryons outside [21,9].

When the DM is WIMP, they can annihilate or decay inside the UCMHs and give signals of gamma-ray, neutrino or cosmic rays. The non-observation of those signals above the background value puts the bound on the size of the primordial perturbation. In the standard model, the constraint on the power spectrum is around $10^{-6}-10^{-8}$ at horizon re-entry in the thermal Universe. However the constraints is limited for the scales larger than the kinetic decoupling scale in the standard Universe.

However in the non-thermal Universe with early matter domination, the constraint can extended to the smaller scales. In this case, the isocurvature perturbation of dark matter WIMP can be generated at small scales and does not damped away even during the kinetic decoupling as we saw in the previous section. The non-observation of the signals can constrain the early matter domination evolution as well as the initial primordial perturbation.

\section{Summary}

The low reheating temperature with WIMP dark matter happens in many theoretical models, including those of the neutralino DM in the low reheating temperature $[17,18]$ and the scenario of decaying heavy particle such as moduli, gravitino [19], or axino [20]. In this models, the large isocurvature perturbation of dark matter can be generated and is not damped away even during the kinetic decoupling.

Furthermore this fact encourages to probe such small scales to extract information about early history of the universe before the BBN. The precise determination of the matter power spectrum can help to construct the early matter domination epoch and probe the time before big bang nucleosynthesis. Therefore the observation of isocurvature perturbation of WIMP dark matter is a great chance to see the non-standard evolution of the early Universe.

The large isocurvature perturbation on small scales of WIMP dark matter can be probed through the formation of of small scale DM clumps [22]. The annihilation of DM in such objects may produce observable signatures of gamma-rays, cosmic rays, or neutrinos [23, 21, 9, 25, 24]. The DM clumps may change also the direct detection rate [26], affect the gravitational wave signals [27], and form the DM stars [28].

\section{Acknlwledgements}

J.G. acknowledges the Max-Planck-Gesellschaft, the Korea Ministry of Education, Science and Technology, Gyeongsangbuk-Do and Pohang City for the support of the Independent Junior Research Group at the Asia Pacific Center for Theoretical Physics. J.G. is also supported by a Starting Grant through the Basic Science Research Program of the National Research Foundation of Korea (No. 2013R1A1A1006701).

\section{References}

[1] P. A. R. Ade et al. [Planck Collaboration], Planck 2013 results. XVI. Cosmological parameters, Astron. Astrophys. 571 (2014) A16 [arXiv:1303.5076 [astro-ph. CO]]. 
[2] M. Y. .Khlopov, A. G. Polnarev and, Primordial Black Holes As A Cosmological Test Of Grand Unification, Phys. Lett. B 97 (1980) 383.

[3] A. M. Green, A. R. Liddle, A. Riotto and, Primordial black hole constraints in cosmologies with early matter domination, Phys. Rev. D 56 (1997) 7559 [astro-ph/9705166].

[4] B. J. Carr, K. Kohri, Y. Sendouda, J. 'i. Yokoyama and, New cosmological constraints on primordial black holes, Phys. Rev. D 81 (2010) 104019 [arXiv:0912.5297 [astro-ph.CO]].

[5] A. S. Josan, A. M. Green, K. A. Malik and, Generalised constraints on the curvature perturbation from primordial black holes, Phys. Rev. D 79 (2009) 103520 [arXiv:0903.3184 [astro-ph.CO]].

[6] A. M. Green, A. R. Liddle and, Constraints on the density perturbation spectrum from primordial black holes, Phys. Rev. D 56 (1997) 6166 [astro-ph/9704251].

[7] D. Jeong, J. Pradler, J. Chluba and M. Kamionkowski, Silk damping at a redshift of a billion: a new limit on small-scale adiabatic perturbations, Phys. Rev. Lett. 113 (2014) 061301 [arXiv:1403.3697 [astro-ph.CO]].

[8] T. Bringmann, P. Scott, Y. Akrami and, Improved constraints on the primordial power spectrum at small scales from ultracompact minihalos, Phys. Rev. D 85 (2012) 125027 [arXiv:1110.2484 [astro-ph.CO]].

[9] T. Bringmann, P. Scott, Y. Akrami and , Improved constraints on the primordial power spectrum at small scales from ultracompact minihalos, Phys. Rev. D 85 (2012) 125027 [arXiv:1110.2484 [astro-ph.CO]].

[10] K. Y. Choi, J. O. Gong and C. S. Shin, WIMP isocurvature perturbation and small scale structure, arXiv:1507.03871 [astro-ph.CO].

[11] S. Hofmann, D. J. Schwarz and H. Stoecker, Damping scales of neutralino cold dark matter, Phys. Rev. D 64 (2001) 083507 [astro-ph/0104173].

[12] A. M. Green, S. Hofmann and D. J. Schwarz, The First wimpy halos, JCAP 0508 (2005) 003 [astro-ph/0503387].

[13] T. Bringmann, Particle Models and the Small-Scale Structure of Dark Matter, New J. Phys. 11 (2009) 105027 [arXiv:0903.0189 [astro-ph.CO]].

[14] W. Hu and N. Sugiyama, Toward understanding CMB anisotropies and their implications, Phys. Rev. D 51 (1995) 2599 [astro-ph/9411008].

[15] P. J. E. Peebles, ApJ, 315:L73 (1987).

[16] B. W. Lee and S. Weinberg, Cosmological Lower Bound on Heavy Neutrino Masses, Phys. Rev. Lett. 39 (1977) 165.

[17] G. B. Gelmini and P. Gondolo, Neutralino with the right cold dark matter abundance in (almost) any supersymmetric model, Phys. Rev. D 74 (2006) 023510 [hep-ph/0602230].

[18] L. Roszkowski, S. Trojanowski and K. Turzy?ski, Neutralino and gravitino dark matter with low reheating temperature, JHEP 1411 (2014) 146 [arXiv:1406.0012 [hep-ph]].

[19] K. Kohri, M. Yamaguchi and J. Yokoyama, Neutralino dark matter from heavy gravitino decay, Phys. Rev. D 72 (2005) 083510 [hep-ph/0502211].

[20] K. Y. Choi, J. E. Kim, H. M. Lee and O. Seto, Neutralino dark matter from heavy axino decay, Phys. Rev. D 77 (2008) 123501 [arXiv:0801.0491 [hep-ph]]. 
[21] M. Ricotti and A. Gould, A New Probe of Dark Matter and High-Energy Universe Using Microlensing, Astrophys. J. 707 (2009) 979 [arXiv:0908.0735 [astro-ph.CO]].

[22] V. S. Berezinsky, V. I. Dokuchaev and Y. N. Eroshenko, Small-scale clumps of dark matter, Phys. Usp. 57 (2014) 1 [Usp. Fiz. Nauk 184 (2014) 3] [arXiv:1405.2204 [astro-ph.HE]].

[23] J. Diemand, B. Moore and J. Stadel, Earth-mass dark-matter haloes as the first structures in the early Universe, Nature 433 (2005) 389 [astro-ph/0501589].

[24] Y. Yang, G. Yang and H. Zong, Neutrino signals from ultracompact minihalos and constraints on the primordial curvature perturbation, Phys. Rev. D 87 (2013) 10, 103525 [arXiv:1305.4213 [astro-ph.CO]].

[25] F. Li, A. L. Erickcek and N. M. Law, A new probe of the small-scale primordial power spectrum: astrometric microlensing by ultracompact minihalos, Phys. Rev. D 86, 043519 (2012) [arXiv:1202.1284 [astro-ph.CO]].

[26] M. Kamionkowski and S. M. Koushiappas, Galactic substructure and direct detection of dark matter, Phys. Rev. D 77 (2008) 103509 [arXiv:0801.3269 [astro-ph]].

[27] A. W. Adams and J. S. Bloom, Direct detection of dark matter with space-based laser interferometers, astro-ph/0405266.

[28] A. V. Gurevich, K. P. Zybin and V. A. Sirota, Small scale structure of dark matter and microlensing, Sov. Phys. Usp. 167 (1997) 913 [astro-ph/9801314]. 